\title{
TH01, a tetrameric short tandem repeat locus in the tyrosine hydroxylase gene: Association with myocardial hypertrophy and death from myocardial infarction?
}

\author{
Michael Klintschar*, Uta-Dorothee Immel, Dankwart Stiller and Manfred Kleiber \\ Institut für Rechtsmedizin, Martin Luther-Universität Halle-Wittenberg, Franzosenweg 1, 06097 Halle/Saale, \\ Germany
}

\begin{abstract}
TH01 is a tetrameric short tandem repeat locus located in intron 01 of the tyrosine hydroxylase gene. The tyrosine hydroxylase catalyzes the hydroxylation of L-tyrosine to L-DOPA and is the rate limiting enzyme in the synthesis of catecholamines like noradrenaline or adrenaline, which are pivotal in the regulation of blood pressure. In a clinical study a strong correlation between alleles $* 9.3$ and $* 10$ and essential hypertension was observed ([2] Hypertension 32: 676-682). To further investigate this association, we typed TH01 in 296 autopsy cases and correlated the genotypes to the heart weight as parameter for myocardial hypertrophy. No significant correlation was observed. Moreover, dividing the studied cases into 2 groups, one including 172 casualties from hypertension-associated diseases (myocardial infarction, left heart failure, aortic aneurysm, spontaneous intracerebral bleeding and cerebral infarction) and one consisting of 124 cases of death unrelated to hypertension, revealed similar allelic frequencies for both groups. Our data thus suggest that TH01 long alleles appear not to lead to a significant increase in the incidence of myocardial hypertrophy or other hypertension associated diseases. This could be explained by a relatively small impact of the TH01 genotype on the blood pressure or by counteraction of another mechanism related to catecholamines and their effect on the human body.
\end{abstract}

\section{Introduction}

TH01 is a tetrameric short tandem repeat (STR) marker located in intron 1 of the tyrosine hydroxylase gene. In clinical studies a correlation between TH01 alleles $* 9.3 / * 10$ and the incidence of manic-depressive illness [1] on one hand, and essential hypertension [2], on the other, was reported. Whereas an association with mental disease was further investigated by other studies [3], this is not the case for the association with raised arterial blood pressure. The goal of the present study was thus to further evaluate potential consequences

*Corresponding author: Michael Klintschar, Institut fur Rechtsmedizin, Martin Luther-University Halle-Wittenberg, Franzosenweg 1, D06112 Germany. Tel.: +49 345557 1591; Fax: +49 345557 1587; E-mail: michael.klintschar@medizin.uni-halle.de. from the reported association with hypertension by testing whether TH01 correlates to morphological consequence of hypertension like left ventricular hypertrophy or an increased risk for myocardial infarction.

\section{Materials and methods}

\subsection{Subjects}

The subjects used for the study were 296 Caucasians who had died from natural causes retrospectively selected from the casework of the Institute of Legal Medicine in Halle, Germany between 1997 and 2003. Of these individuals, 172 had died from causes that are generally assumed to be closely associated with hy- 
Table 1

Causes of death according to the ICD-10 classification (simplified) in the 296 cases studied. In 172 of these cases the cause of death was a disease for which hypertension is a well recognized risk factor. For 124 causes the cause of death was without association with hypertension

\begin{tabular}{lr}
\hline \multicolumn{2}{l}{ Causes of death without significant association to hypertension } \\
\hline Infectious and parasitic diseases & 2 \\
Neoplasms & 54 \\
Disorders of blood, blood-forming organs and the immune & 2 \\
system & \\
Endocrine, alimentary and metabolic disorders & 8 \\
Mental and behaviour disorders & 2 \\
Disorders of the nervous system & 5 \\
Other disorders of the circulatory system & 15 \\
Disorders of the respiratory system & 19 \\
Disorders of the gastrointestinal system & 14 \\
Other disorders & 3 \\
Causes of death associated to hypertension & \\
Myocardial Infarction & 128 \\
Ruptured aortic aneurysm & 4 \\
Cerebral Infarction or spontaneous bleeding & 20 \\
Left heart failure & 20 \\
\hline
\end{tabular}

pertension. In the remaining 124 cases other natural causes were established (Table 1).

To ensure that the groups reflect the spectrum of causes of death in a German population, the cases included were selected to match the distribution of causes of death according to the International Classification of Diseases (ICD-10) given in the deaths statistic of the Federal Republic of Germany for 2000 (http://www.destatis.de/presse/deutsch/pm2001/ p3840092.htm). The age range was comparable for both groups (19-98 years; mean 67; SD 15 for the "hypertension associated" group and 21-100; mean 64; SD 16) for the "non hypertension associated" group). Both groups had similar gender distributions (48\% and $49 \%$ females, respectively).

\subsection{Genotyping}

DNA was isolated from whole blood by a modified alkaline lysis protocol [4]. Genotypes for the TH01 polymorphism were in part determined by PCR followed by native PAGE and silver staining as described [5] in part by capillary electrophoresis (ABI 310 Genetic Analyzer, ABI, Foster City, CA.) and commercially available PCR kits as described [6].

\subsection{Statistical analyses}

$\chi^{2}$ analysis for deviation from Hardy-Weinberg equilibrium and population heterogeneity was performed using the RxC software by G. Carmody, the Genepop software (http://wbiomed.curtin.edu.au/genepop/) and custom made Microsoft Excel ${ }^{\circledR}$ Spreadsheets.
Table 2

Allelic frequencies for TH01 in 172 deaths due to diseases related to hypertension ("BP related") and 124 deaths due to diseases without strong relation to hypertension ("non BP related")

\begin{tabular}{ccc}
\hline Allele & BP related & Non BP related \\
\hline 5 & $0.7 \%$ & $0.4 \%$ \\
6 & $21.3 \%$ & $19.7 \%$ \\
7 & $15.8 \%$ & $17.2 \%$ \\
8 & $10.3 \%$ & $10.9 \%$ \\
9 & $18.4 \%$ & $17.5 \%$ \\
9.3 & $33.1 \%$ & $33.6 \%$ \\
10 & $0.4 \%$ & $0.7 \%$ \\
\hline
\end{tabular}

Table 3

Mean weight of the heart at autopsy and pooled TH01 genotypes. (SD: Standard deviation). The genotypes were pooled as follows: small/small: Genotypes without a $* 9.3$ or $* 10$ allele. Small/Long: Genotypes with one $* 9.3$ or $* 10$ allele. Long/Long: Genotypes with two $* 9.3$ or $* 10$ alleles

\begin{tabular}{rccc}
\hline & Small/small & Small/long & Long/long \\
\hline Weight of the heart (g) & & & \\
Mean & 422 & 418 & 424 \\
SD & 124 & 121 & 101 \\
\hline
\end{tabular}

\section{Results}

Genotypes were assigned on the basis of the pattern of bands on electrophoretic gels according to recommendations of the ISFG based on the number of repeat units [7]. TH01 has at least 6 alleles with 5 to $* 10$ complete (TCAT) repeats and a common allele "*9.3" with 9 complete repeats and one incomplete (TCA) repeat [8]. Hardy-Weinberg expectations were met.

Dividing the cases into those with major association with hypertension and those without major association (Table 1) led to the formation of two groups which were similar in respect to the allelic frequencies in a $\chi^{2}$ Test (Table 2).

As phenotypic effects are only assumed for the "long" alleles $* 9.3$ or $* 10$, genotypes were pooled into 3 groups: Those without alleles $* 9.3$ or $* 10$ (small/small), those with one allele $* 9.3$ or $* 10$ (small/long) and those with two alleles $* 9.3$ or $* 10$ (long/long). As shown in Fig. 2, the distribution of pooled genotypes was similar for both groups. Moreover, the mean weight of the hearts at autopsy is independent from the pooled genotype (Table 3 ).

\section{Discussion}

Unlike for many STR loci, for TH01 the exact location (intron 01 of the tyrosine hydroxylase gene) and adjacent genes (Harvey ras 1 oncogene and insulin gene) are known [2]. The tyrosine hydroxylase is the 


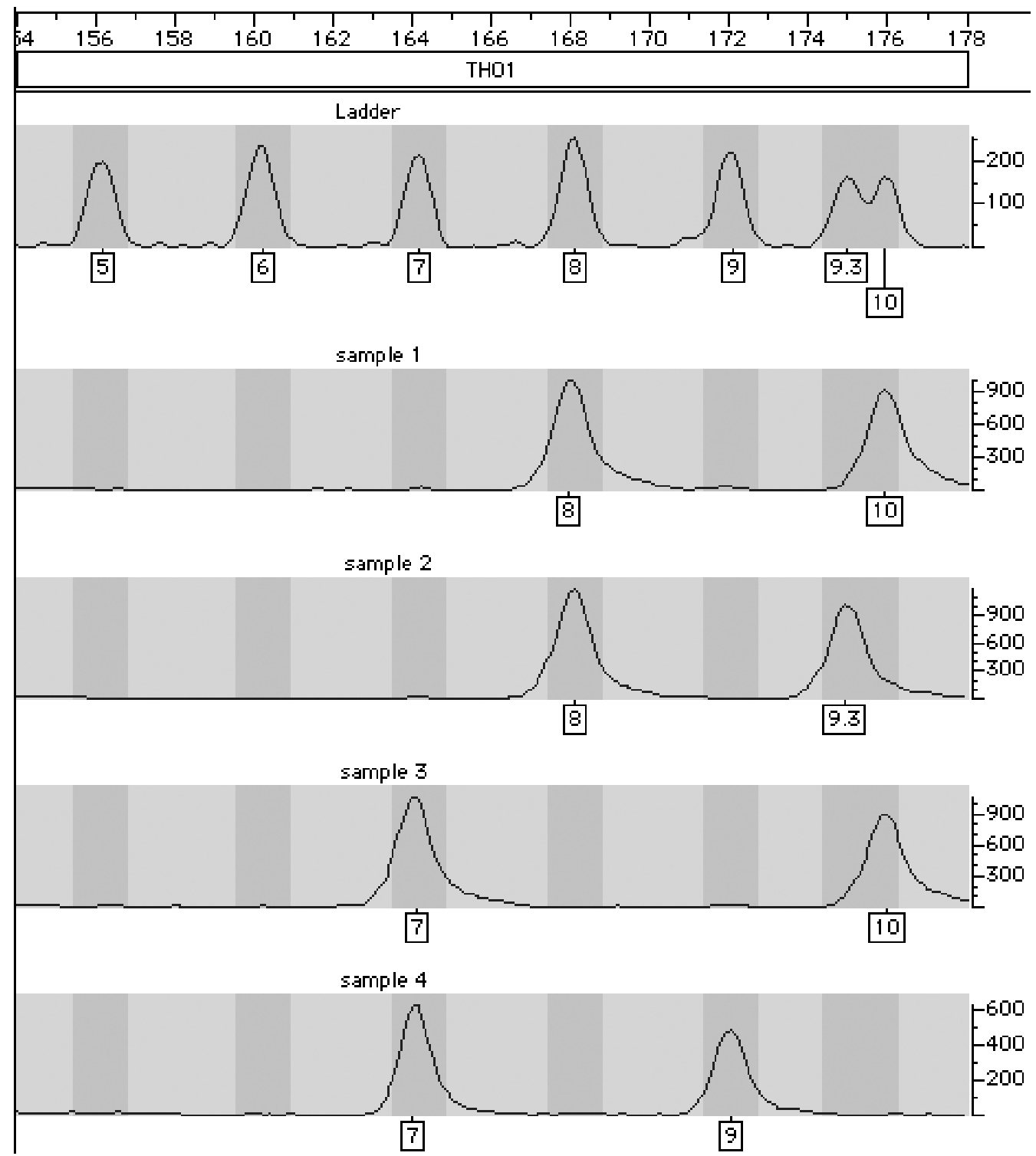

Fig. 1. Pherogram for the TH01 locus on the ABI 310 Genetic Analyzer. Upper panel: Allelic ladder with alleles 5 to $9,{ }^{*} 9.3$ and ${ }^{*} 10$ in the range of 156 to $176 \mathrm{bp}$. The 4 lower panels show 4 heterozygote samples $(8,10 ; 8,9.3 ; 7,10$ and 7,9$)$.

rate limiting enzyme in the synthesis of catecholamines like noradrenaline. Catecholamines act as neurotransmitters and hormones and are pivotal in the regulation of blood pressure [9]. In a clinical study on British subjects a positive association of allele "E" (correponding to alleles $* 9.3$ and $* 10$ in a repeat based nomenclature) with the prevalence of essential hypertension was found, whereas the allele "D" (corresponding to allele 9) appeared to occur more often in a normotensive than in a hypertensive group [2].

This association was established despite TH01 being a non-coding marker. Nevertheless, there are two indirect ways in which this marker could exert influence on the phenotype of an individual: Firstly it could be in close LD to a gene variant associated with hypertension: One fact fostering this possibility is the extremely low rate of spontaneous repeat array mutations of this marker compared to other STRs leading to conservation of LD due to a genetic bottleneck [8].

The second possibility would be a functional activity of the TH01 sequence via gene regulation or other, hitherto not sufficiently investigated mechanisms [10]. 


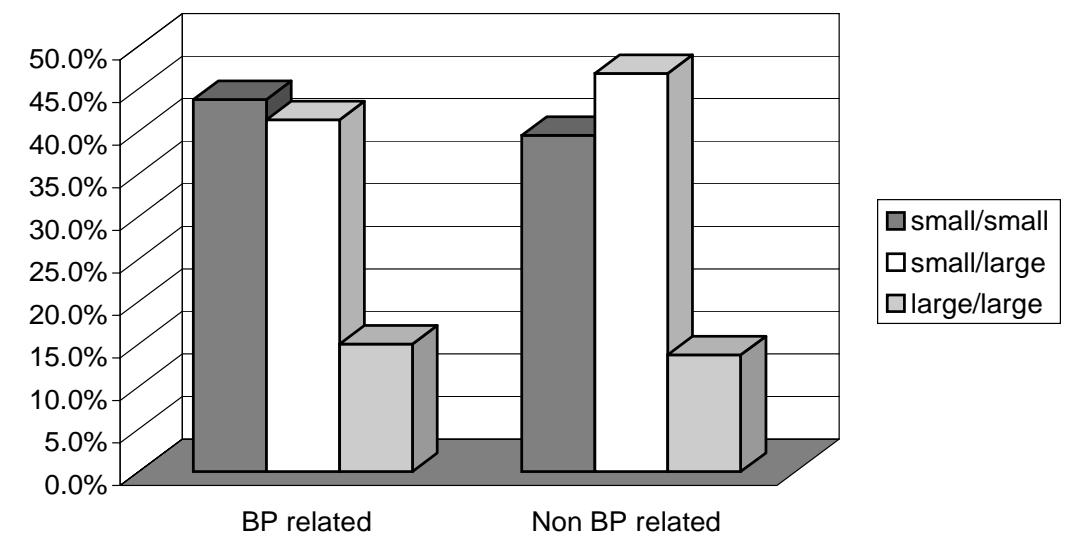

Fig. 2. Pooled genotypes for TH01 in 172 deaths due to diseases closely related to hypertension ("BP related") and 124 deaths due to diseases without strong relation to hypertension ("non BP related", as outlined in Table 1). The genotypes were pooled as follows: small/small: Genotypes without a ${ }^{*} 9.3$ or $^{*} 10$ allele. Small/Long: Genotypes with one ${ }^{*} 9.3$ or ${ }^{*} 10$ allele. Long/Long: Genotypes with two ${ }^{*} 9.3$ or $* 10$ alleles. No significant differences were found between both groups.

This possibility is supported by recent in vitro investigations which proved that TH01 acts as a quantitative genomic effector modulating the activity of the $\mathrm{TH}$ gene: The TH01 locus inhibits transcription of the tyrosine hydroxylase gene, and the inhibition is proportional to the number of repeats [11]. It is assumed that the effect on transcription is specifically due to the repeat sequence by interaction with factors such as ZNF191 binding to the (TCAT)n repeat, and that other STRs with this repeat motif might have similar effects on other genes. Nevertheless it remains to be established whether such STRs influence transcription in vivo.

From the pathological point of view, chronically raised arterial blood pressure in essential hypertension leads to myocardial hypertrophy and thus increased heart weight upon autopsy. Moreover, it is a risk factor for several potentially lethal events such as myocardial infarction, aortic aneurysm, left heart failure, spontaneous intracerebral bleeding and cerebral infarction. Although there are numerous other risk factors known, a genetic finding associated with substantially increased blood pressure can thus be expected to result in an increased incidence of those events. On the other hand, other causes of death, like inflammatory diseases or carcinomas, are assumed to be without significant correlation to hypertension. In our study we found neither a correlation of TH01 genotypes and heart weights in autopsy cases (Table 2), nor differences in TH01 genotypes between casualties from hypertension related events and deaths unrelated to hypertension. (Fig. 1).

The correlation to hypertension reported by Sharma et al. [2] made us initially expect an influence of TH01 on heart weight and also a higher incidence of hypertension related causes of death in persons with $* 9.3$ and *10. In part the lack of these findings might be explained by the fact that in the latter study no discrimination between $* 9.3$ and $* 10$, two alleles which are difficult to separate, was performed. Nevertheless, as $* 10$ is rare compared to $* 9.3$ (with a typical ratio of 1:20-30 in Caucasians [12], this should have only minor impact on an association study. Another explanation would be that the association with blood pressure is too weak to cause significant increased myocardial hypertrophy in a sample of less than 300 individuals, or that the formation of myocardial hypertrophy is inhibited by other, hitherto unknown mechanisms.

In conclusion our findings suggest that TH01 neither influences the prevalence of myocardial hypertrophy nor the distribution of causes of death in the sample studied herein. Despite the reported correlation to hypertension, this marker thus appears not to be associated with diseases secondary to this status.

\section{Acknowledgements}

The authors would like to thank A. Graul, A. Heide and S. Salostowitz for excellent technical assistance.

\section{References}

[1] R. Meloni, M. Leboyer, F. Bellivier, B. Barbe, D. Samolyk, J.F. Allihaire and J. Mallet, Association of manic-depressive illness with tyrosine hydroxylase microsatellite marker, Lancet 345 (1995), 932. 
[2] P. Sharma, A. Hingorani, H. Jia, M. Ashby, R. Hopper, D. Clayton and M.J. Brown, Positive association of tyrosine hydroxylase microsatellite marker to essential hypertension, $\mathrm{Hy}$ pertension 32 (1998), 676-682.

[3] R.D. Todd, E.A. Lobos, A. Parsian, S. Simpson and J.R. DePaulo, Manic-depressive illness and tyrosine hydroxylase markers, Lancet 347 (1996), 1634.

[4] M. Klintschar and F. Neuhuber, Evaluation of an Alkaline Lysis Method for the Extraction of DNA from Whole Blood and Forensic Stains for STR Analysis, J For Sci 45 (2000), 669-673.

[5] M. Klintschar and M. Kubat, A study of the short tandem repeat systems HUMVWA and HUMTH01 in an Austrian population sample, Int J Legal Med 107 (1995), 329-330.

[6] B. Reichenpfader, U. Immel and M. Klintschar, Population data on the AmpFLSTR plus PCR amplification kit in Germans and Austrians, Forensic Sci Int 132 (2003), 84-86.

[7] W. Bar, B. Brinkmann, B. Budowle, A. Carracedo, P. Gill, P. Lincoln, W.R. Mayr and B. Olaisen, DNA recommendations. Further report of the DNA Commission of the ISFH regarding the use of short tandem repeat systems. International Society for Forensic Haemogenetics, Int J Legal Med 110 (1997), 175-176.

[8] B. Brinkmann, M. Klintschar, F. Neuhuber, J. Huhne and B. Rolf, Mutation rate in human microsatellites: influence of the structure and length of the tandem repeat, Am J Hum Genet 62 (1998), 1408-1415.

[9] K.H. Rahn, M. Barenbrock and M. Hausberg, The sympathetic nervous system in the pathogenesis of hypertension, $J$ Hypertens 3 (1999), 11-14.

[10] Y. Kashi, D. King and M. Soller, Simple sequence repeats as a source of quantitative genetic variation, Trends Genet $\mathbf{1 3}$ (1997), 74-78.

[11] V. Albanese, N.F. Biguet, H. Kiefer, E. Bayard, J. Mallet and R. Meloni, Quantitative effects on gene silencing by allelic variation at a tetranucleotide microsatellite, Hum Mol Genet 10 (2001), 1785-1792.

[12] B. Brinkmann, A. Sajantila, H. Goedde, H. Matsumoto, K. Nishi and P. Wiegand, Population genetic comparisons among eight populations using allele frequency and sequence data from three microsatellite loci, Eur J Hum Genet 4 (1996), $175-182$. 


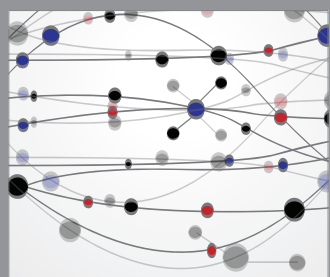

The Scientific World Journal
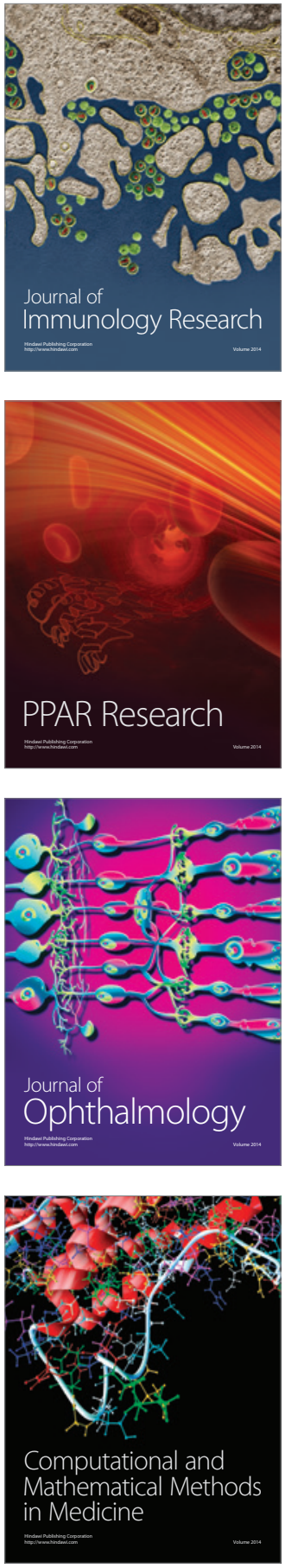

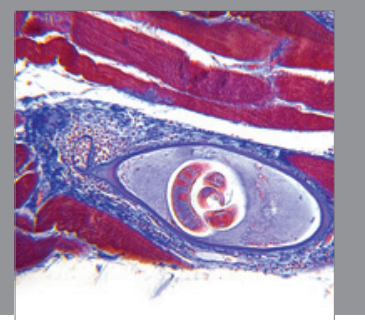

Gastroenterology

Research and Practice
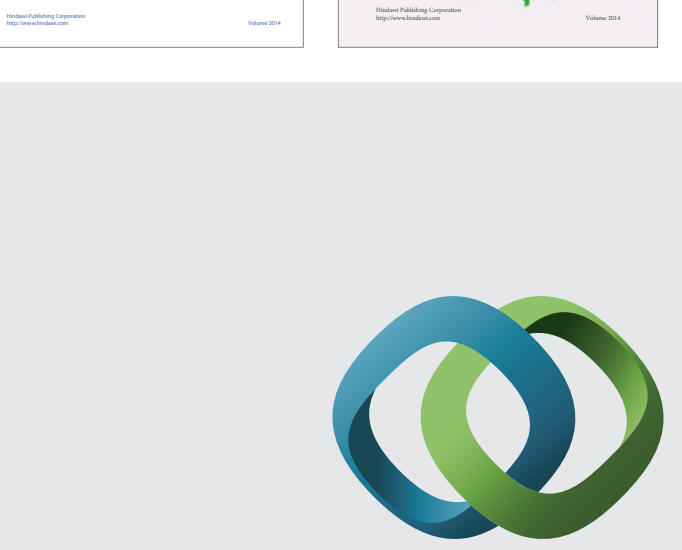

\section{Hindawi}

Submit your manuscripts at

http://www.hindawi.com
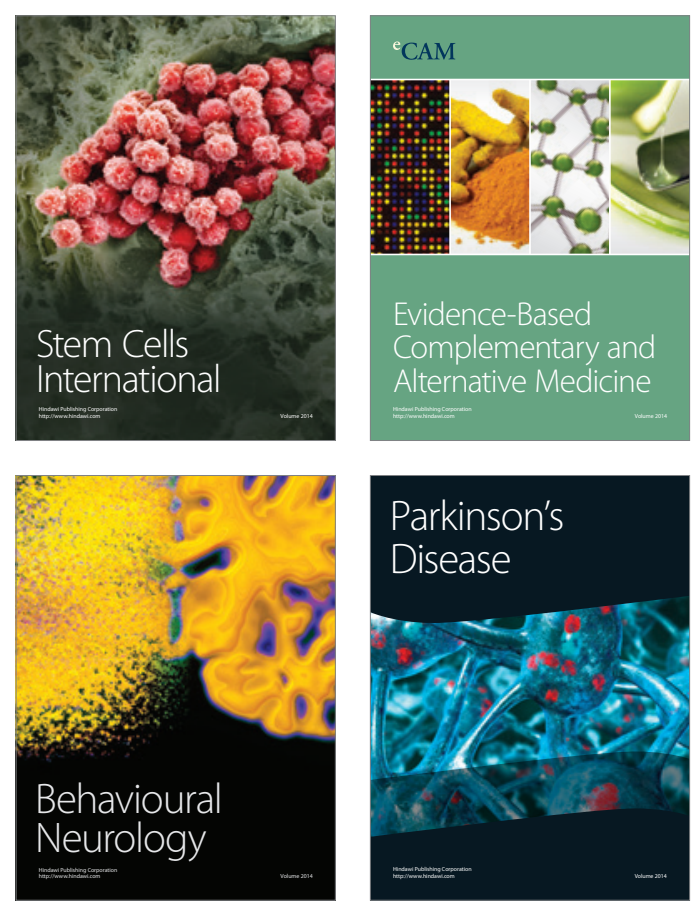

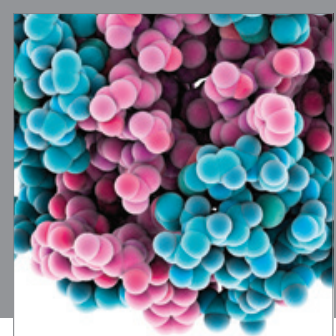

Journal of
Diabetes Research

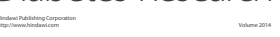

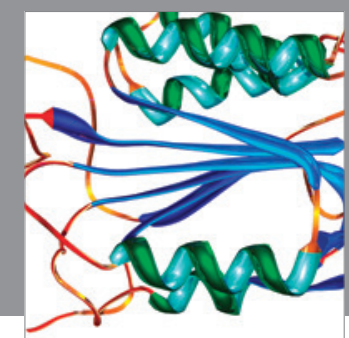

Disease Markers
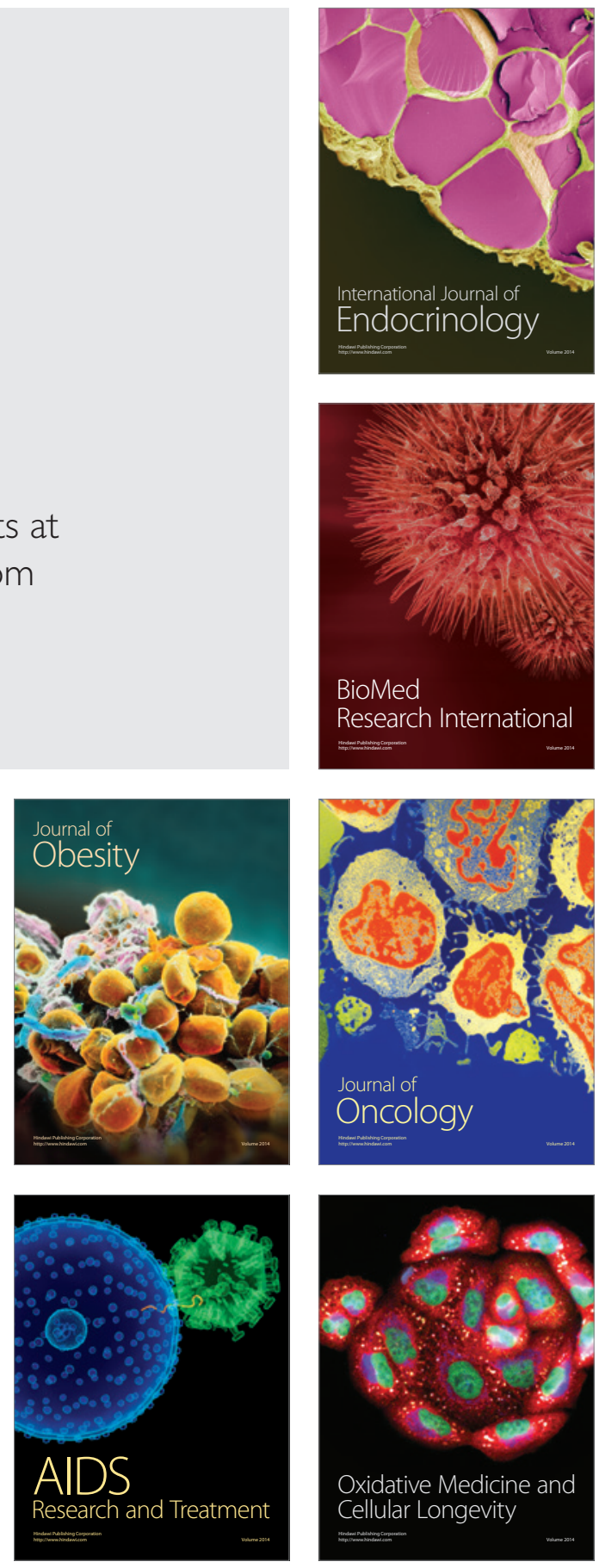